\title{
Prophylactic and therapeutic DNA vaccines against Chagas disease
}

\author{
Minerva Arce-Fonseca, Martha Rios-Castro, Silvia del Carmen Carrillo-Sánchez, Mariana Martínez-Cruz \\ and Olivia Rodríguez-Morales*
}

\begin{abstract}
Chagas disease is a zoonosis caused by Trypanosoma cruzi in which the most affected organ is the heart. Conventional chemotherapy has a very low effectiveness; despite recent efforts, there is currently no better or more effective treatment available. DNA vaccines provide a new alternative for both prevention and treatment of a variety of infectious disorders, including Chagas disease. Recombinant DNA technology has allowed some vaccines to be developed using recombinant proteins or virus-like particles capable of inducing both a humoral and cellular specific immune response. This type of immunization has been successfully used in preclinical studies and there are diverse models for viral, bacterial and/or parasitic diseases, allergies, tumors and other diseases. Therefore, several research groups have been given the task of designing a DNA vaccine against experimental infection with T. cruzi. In this review we explain what DNA vaccines are and the most recent studies that have been done to develop them with prophylactic or therapeutic purposes against Chagas disease.
\end{abstract}

Keywords: Chagas disease, Trypanosoma cruzi, DNA vaccines

\section{Introduction}

Chagas disease is a zoonosis caused by Trypanosoma cruzi in which the most affected organ is the heart. It is estimated that about 10 million people are infected with the parasite in the Americas and 25 million more are at risk of contracting the disease [1]. The effectiveness of conventional chemotherapy is very low; treatment is successful in $55.8 \%$ of cases of children with early infection. Most individuals in the chronic phase are resistant to treatment with existing drugs and carry the infection for their lifetime [2-4]. Despite recent efforts, there is currently no better or effective treatment available [5]. DNA vaccines provide an alternative for both prevention and treatment of a variety of infectious diseases, including Chagas disease [6-8].

\section{Review}

\section{DNA vaccines}

In recent years, tools of molecular biology and understanding of the mechanisms of the immune response have

\footnotetext{
*Correspondence: rm.olivia@gmail.com

Department of Molecular Biology, Laboratory of Molecular Immunology and Proteomics. Instituto Nacional de Cardiología "Ignacio Chávez", Juan Badiano No. 1, Col. Sección XVI, Tlalpan, C.P. 14080 Mexico City, Mexico
}

helped to expand the development of vaccines. It has been found necessary, in the case of many diseases, to produce vaccines that induce a response by cytotoxic $\mathrm{T}$ lymphocytes (CTL). The live attenuated virus vaccines are able to induce both CTL and humoral responses; however, the inactivated virus and the recombinant proteins generally do not induce a potent CTL response. The vaccines from live attenuated organisms provide broad protection, although security for certain diseases may be controversial, such as acquired immunodeficiency syndrome because of the potential risk of reversion to virulence through mutation or recombination with a wild pathogen [9].

Recombinant DNA technology has allowed the creation of some vaccines using recombinant proteins or virus-like particles. These vaccines are considered safe and able to induce an efficient immune response $[9,10]$.

A DNA vaccine can be defined as a plasmid containing a viral, bacterial or parasitic gene that can be expressed in mammalian cells (in the case of infectious diseases) [11,12] or a gene encoding a mammalian protein (in the case of non-infectious diseases such as cancer [13-15], autoimmunity [16-18] and allergies [19-21]). The amount of plasmid that is internalized in vivo has been estimated in the picogram range after injection into mouse muscle [22] 
and in the range of picograms to femtograms after 1 to 7 days of intravenous administration of DNA complexes with cationic lipids [23]. Because the plasmids do not replicate in mammalian cells, the amount available for the gene expression is very low. Therefore, a strong promoter/ terminator to drive the expression of the gene must be chosen [9]. Several studies have shown that the amount of antigen produced after inoculation of the DNA is in the range of picograms to nanograms in vivo. Plasmid DNA is usually inoculated into the host or animal model by direct injection into the muscle but there are other inoculation routes. The nucleic acid is taken up and expressed by the host cells, causing the subsequent induction of both humoral and cellular specific immune responses [9,10,24]. This type of immunization has been successfully used in preclinical studies and there are diverse models for viral, bacterial and/or parasitic diseases, allergies, tumors and other diseases [24,25]. In recent years, the development of vaccines against parasites has had a significant progress in which a variety of animal models and routes of administration have been tested [26-28]; regarding Chagas disease, several research groups have been given the task of designing a DNA vaccine against experimental infection with T. cruzi (Table 1).

\section{DNA vaccines against Chagas disease}

- Trans-sialidase family and surface proteins antigens

Garg and Tarleton immunized C57BL/6 mice with ASP-1, ASP-2 and TSA-1 genes of the trans-sialidase family and obtained few antibodies and CTL low activity. Protection against lethal challenge was $30 \%$ to $60 \%$. When the interleukin-12 (IL-12) and granulocyte macrophage colony-stimulating factor (GM-CSF) genes were coadministered CTL activity, antibody production and resistance to infection with the parasite were increased. Lower parasitism in the tissues analyzed, less swelling and less damage in skeletal muscle were also observed. Survival to the challenge was $60 \%$ to $80 \%$; greater protection by the mixture of the three genes was not generated [29].These results show that trans-sialidases, remain good candidates for vaccines, but they are a polymorphic gene family and response may vary accordingly. Co-administration of cytokine genes enhances the immune response.

The Boscardin group selected an amastigote surface protein 2 (ASP-2) related-gene of $T$. cruzi which is an antigen recognized by antibodies and $T$ cells from infected mice and humans. Four sets of genes were amplified using specific primers for the asp-2 gene from amastigotes cDNA of T.cruzi Y strain and a clone with the highest degree of identity for the gene of interest was selected, this gene was named clone 9. BALB/c mice immunized with a plasmid containing clone 9 gene produced specific antibodies and $\mathrm{CD}^{+} \mathrm{T}$ cells secreting IFN- $\gamma$. After trypomastigote challenge a reduction in and increased survival in the animals was observed [30]. The use of parasite surface proteins, secreted proteins or proteins that can be expressed on the infected cell surface are also of interest to identify their possible role as important immunogens for protective induction.

Table 1 DNA vaccines containing Trypanosoma cruzi genes for prophylactic use

\begin{tabular}{|c|c|c|c|c|c|}
\hline Vector & Model & Immune Response & Survival (\%) & Recombinant antigen & Reference \\
\hline pCMVI.UBF3/2 & $\mathrm{C} 57 \mathrm{BL} / 6$ & Th1 & $30-60$ & ASP-1, ASP-2 and TSA-1 & 29 \\
\hline pcDNA3 & $\mathrm{BALB} / \mathrm{C}$ & Th1 & 100 & Clone 9 (ASP-2) & 30 \\
\hline \multirow[t]{2}{*}{ pCMV } & $\mathrm{BALB} / \mathrm{C}$ & ND & $25-100$ & TSSA & 31 \\
\hline & C57BL/6 & & & & \\
\hline pcDNA3 & $\mathrm{BALB} / \mathrm{C}$ & Th1 & ND & catalytic domain of a trans-sialidase & 32 \\
\hline pcDNA3.1 & $\mathrm{C} 57 \mathrm{BL} / 6$ & Th1 & ND & TcG1, TcG2 and TcG4 & 33 \\
\hline $\mathrm{pBK}-\mathrm{CMV}$ & $B A L B / C$ & Th1/Th2 & 92 & TCSP & 35 \\
\hline pBK-CMV & $\mathrm{BALB} / \mathrm{C}$ & Th1 & 75 & TCSSP4 & 36 \\
\hline pBK-CMV & Beagle dogs & Th1 & 100 & TCSP, TCSSP4 & 37 \\
\hline pSCREEN & $\mathrm{BALB} / \mathrm{C}$ & Th1 & ND & Cruzipain & 40 \\
\hline pcDNA3 & $\mathrm{C} 3 \mathrm{H} / \mathrm{HeN}$ & Th1 & 100 & Cruzipain & 41 \\
\hline pCMV & C57BL/6J & Th1 & 80 & LYT1 & 42 \\
\hline Vaccinia Virus & $\mathrm{BALB} / \mathrm{C}$ & Th1 & $25-50$ & ANYNFTLV epitope & 43 \\
\hline pcDNA3.1 & $\mathrm{BALB} / \mathrm{C}$ & ND & 70 & Tc13 & 44 \\
\hline pCMV4 & $\mathrm{BALB} / \mathrm{C}$ and $\mathrm{C} 57 \mathrm{BL} / 6$ transgenic mice & Th1 & 75 & PFR2 and PFR3 & 45 \\
\hline pcDNA3.1 & Holtzman rats & Th1/Th2 & ND & CCL4/MIP-1 beta chemokine & 46 \\
\hline
\end{tabular}

$\mathrm{ND}=$ Not determined. 
Miyahira and colleagues examined the role of natural killer (NK) T cells against T. cruzi infection; they infected CD1d-deficient mice with blood trypomastigotes of Tulahuén strain and they found that the absence of NK cells did not increase susceptibility to infection, comparison of the parasitaemia and survival of these animals with normal BALB/C and C57BL mice being very similar. Subsequently, they analyzed whether administration of $\alpha$-galactosylceramide $(\alpha$-GalCer) conferred protection, resulting in a change in parasitaemia only in the late stage of infection, and a slight improvement in survival rate when mice were infected intraperitoneally. The combined use of $\alpha-\mathrm{GalCer}$ and benznidazole did not increase the therapeutic efficacy of the drug, suggesting that NK T cells do not play a major role in resistance to infection. In order to determine whether NKcells involved in the immunity induced by DNA immunization, animals were immunized with the pTSSA-plasmid, which encodes a T. cruzi trans-sialidase. It was found that parasitaemia was suppressed and survival in infected animals consequently increased in those that were plasmidimmunized, in comparison to animals immunized with the vector alone. Co-administration of $\alpha$-GalCer and pTSSAplasmid immunization impaired the protective effect induced by theDNA, as the parasiatemia was increased and did not improve survival compared to mice immunized only with the empty plasmid. When mice were immunized with DNA encoding a $\mathrm{CD} 8^{+} \mathrm{T}$ cells epitope and $\alpha-\mathrm{GalCer}$ was co-administered they showed a much lower $\mathrm{CD} 8^{+}$ $\mathrm{T}$ cell production than those animals that were only immunized [31]. This study demonstrates that the induction and activation of NK T cells can have adverse effects on the protective immunity induced by DNA vaccination, possibly because NK cells have Toll-like receptors capable of recognizing parasite-infected cells and probably transfected cells are deleted and thereby the activation of immune response is decreased.

Mussalem et al. used Propionibacterium acnes or a soluble polysaccharide extracted from the bacterial wall to modulate the immune response induced by the $\mathrm{p} 154 /$ 13 plasmid containing a gene coding for the catalytic domain of a T. cruzi trans-sialidase. Treatment with these adjuvants decreased the parasitaemia peak and increased the Th1 specific immune response toward the transsialidase as was observed by a low IgG1/IgG2a ratio and in vitro strong synthesis of IFN- $\gamma$ by $\mathrm{CD} 4 \mathrm{~T}$ cells [32]. These data suggest that adjuvants can improve the DNA vaccines protection against $T$. cruzi.

Bathia and Garg vaccined C57BL/6 mice with three doses of DNA plasmid encoding TcG1, TcG2 and TcG4 antigens which are expressed in the plasma membrane of trypomastigotes/amastigotes and also with plasmids encoding IL-12 and GM-CSF. IgG2b/IgG1 isotypes were associated with $50 \%-90 \%$ of tissue parasite load control in the acute stage and with an undetectable parasite level during the chronic stage. Serum and cardiac IFN- $\gamma$, tumor necrosis factor (TNF) levels and the heart inflammatory infiltrate decreased in vaccinated mice during the development of chronic disease [33]. Together, these results demonstrated the identification of new vaccine candidates that provide protection against $T$. cruzi in experimental mice.

Recently, the Carlos Slim Health Institute proposed an important initiative to accelerate the development of a new bivalent vaccine comprising two $T$. cruzi recombinant antigens, Tc24 and TSA-1, against Chagas disease in Mexico. A consortium including the Centro de Investigación y de Estudios Avanzados of the Instituto Politécnico Nacional (CINVESTAV-IPN), Laboratorios de Biológicos y Reactivos de México, S. A. de C. V. (BIRMEX) in Mexico City, and the Centro de Investigaciones Regionales "Dr. Hideyo Noguchi" in Mérida, state of Yucatán was formed. This initiative will work together with a focus on developing an optimal vaccine in the least possible time [34].

The Rosales-Encina group immunized BALB/c mice with the $T c S P$ gene encoding a member of the trans-sialidase family, TcSP4 gene or the recombinant proteins. The serum cytokine analysis showed that immunization with the recombinant protein or with TcSP resulted in a T cell mixed Th1-Th2 response. IFN- $\gamma$ was detected in the TcSP4 gene vaccinated-mice sera shortly after immunization, suggesting a Th1 response. The immunized mice were infected with the T. cruzi H8 strain blood trypomastigotes. Only mice immunized with DNA showed a significant reduction in the peak parasitaemia and lethal challenge survival $[35,36]$. These studies demonstrate that DNA vaccination induces a protective immune response in contrast to that produced by the homologous recombinant protein during T. cruzi experimental infection.

TcSP and TcSSP4 genes were also tested prophylactically in a canine model of Chagas disease; the antibody analysis revealed that the dominant subclass was IgG2. Immunization with both recombinant plasmids induced cell mediated immunity characterized by lymph proliferation and IFN- $\gamma$ production [37]. In these same animals, immunization decreased the quality and quantity of electrocardiographic changes, thereby preventing progression to more serious cardiac disorders [38]. A partial protective effect for the prevention of macroscopic and microscopic damage in cardiac tissue during the chronic phase was also observed [39]. Two T. cruzi genes generating a moderate level of protection in the chronic phase of the disease were proposed.

\section{- Antigenic proteins}

Another T. cruzi antigen that has been studied by researchers is cruzipain $(\mathrm{Cz})$. It has been shown that this 
protein is antigenic both in humans and in mice during parasite infection. Schanpp et al. immunized mice with the $\mathrm{Cz}$ gene and showed a CTL response capable of recognizing and lysing cells infected with T. cruzi [40]. Some antigens may be immunogenic and induce protection using recombinant protein or DNA vaccination, such as the $\mathrm{Cz}$ gene, which is a good candidate antigen for the development of an anti-T. cruzi vaccine. $\mathrm{Cz}$ is an enzyme that can be secreted by the parasite and it is considered a virulence factor so if it can be blocked or recognized on the surface of infected cells by CTL then will be a good vaccine candidate, as was observed in this study.

Cazorla et al. evaluated mucosal immunization in $\mathrm{C} 3 \mathrm{H} /$ HeN mice which received Salmonella carrying a plasmid coding for the $\mathrm{Cz}(\mathrm{SCz})$ together with bacteria containing a GM-CSF encoding plasmid. As an additional strategy, prime-boost protocols were established in which mice were vaccinated with $\mathrm{SCz}$ and were subsequently boosted with recombinant $\mathrm{Cz}(\mathrm{rCz})$ co-administered with different adjuvants, such as CpG-ODN and MALP-2 motifs. Protocols of four $\mathrm{SCz}$ oral doses induced a mucosal immune response, mainly characterized by the secretion of immunoglobulin A (IgA) and cell proliferation of gut-associated lymphoid tissues, with a weak systemic immune response. In contrast, the protocol including a boost with $\mathrm{rCZ}$ and CpG-ODN produced a strong systemic immune response reflected in the titres of specific IgG against $\mathrm{Cz}$, splenocyte proliferation, interferon-gamma (IFN- $\gamma$ ) secretion, and delayed hypersensitivity response. The challenge of vaccinated mice resulted in significantly lower levels of parasitaemia. Parasite control was also evident from the reduction of tissue damage [41]. The results demonstrate that administration of Salmonella-mediated Cz-DNA alone or in combination with CpG-ODN or $\mathrm{rCz}$ and MALP-2 promotes the immune response to control both the infection with $T$. cruzi and the collateral damage to muscle tissue.

Fralish and Tarleton performed the screening of a cDNA expression library of $T$. cruzi amastigote with monoclonal anti-amastigote antibodies and identified the $F C a B P$ gene encoding the flagellar $\mathrm{Ca}\left(2^{+}\right)$binding protein, Tc $\beta 3$ gene encoding a new homologue of the adaptin AP-3 complex $\beta 3$ subunit and LYT1 gene which encodes a secreted T. cruzi protein involved in cell lysis and infectivity. The three gene peptides induced a response of cytotoxic $\mathrm{T}$ cells in chronically infected mice; however, the immunization with only LYT1 gene protected $80 \%$ of mice from $T$. cruzi lethal challenge. Alternatively gene mixtures were tested in immunization assays [42]. This study demonstrates that the ability of $T$. cruzi proteins to induce immune responses in infected hosts, is not necessarily associated with the capacity to induce protection and thus the products of the individual genes and multigene families can serve as effective vaccines.

The Miyahira group used a recombinant adenovirus and the vaccinia virus as vectors expressing a $\mathrm{CD} 8^{+} \mathrm{T}$ cells epitope (ANYNFTLV), which is derived from a $T$. cruzi antigen. The immunization with adenovirus and the vaccinia virus booster was effective in inducing a cellular immune response and consequently protected mice from parasite lethal challenge. The response was increased by co-administration of recombinant vaccinia virus expressing the receptor of the nuclear factor kappa-lightchain-enhancer of activated $\mathrm{B}$ cells $(\mathrm{NFKB})$ ligand as adjuvant [43]. The results of this group are spectacular identifying an eight amino acid region of a molecule able to induce a sufficient immune response to control T. cruzi lethal infection, but again keep in mind that the peptides to CD4T or CD8T depend on the type of $\mathrm{MHC}$ for its proper recognition.

García et al. did not find antibodies when DNA plasmid containing the gene of the Tc13 antigen of the T. cruzi Tulahuén strain ( $\mathrm{Tc} 13 \mathrm{Tul}$ ) was administered to BALB/c mice; however, vaccination induced specific memory $\mathrm{T}$ cells with no IFN- $\gamma$ production. From $40 \%$ to $80 \%$ of the mice immunized showed signs of hepatotoxicity and heart, liver and spleen changes five months post-immunization. After challenge, a significant reduction in parasiatemia during the acute stage was observed without modification in the survival rate. The immunization resulted in decreased severity of cardiac damage in the chronic stage [44]. Although in this study it is reported that the chosen antigen induces protection in the chronic stage of the disease, hepatotoxicity, as well as heart and spleen damage were also demonstrated. It is very important that chosen vaccination antigens are completely safe and they induce a significant level of protection.

Morell et al. immunized mice with DNA containing genes forT. cruzi paraflagelar rod proteins (PFR2 and PFR3) alone or fused to the 70 kilodalton heat shock protein (HSP70). Both immunizations induced high levels of antiPFRs IgG2a; however, only the PFR2-HSP70 immunization induced a significant increase in the IL-12 and IFN- $\gamma$ expression and a decrease in the percentage of cells expressing IL-4 [45]. The chimerical gene immunization may provide a protective response against $T$. cruzi experimental infection.

\section{- Non-specific T. cruzi proteins}

Roffe et al. injected a cardio-toxin and four doses of $100 \mu \mathrm{g}$ of plasmid encoding for the chemokine (C-C motif) ligand 4/macrophage inflammatory protein 1 beta (CCL4/MIP-1 beta) into rats intramuscularly. After 14 days after the last immunization the rats were challenged with T. cruzi CL-Brener clone. The myocarditis 
was still intense at day 30 but the inflammatory infiltrate showed a focal distribution. Increased anti-CCL4/MIP1beta levels in the T. cruzi infected animals were induced by the immunization. This was associated with an exacerbation of inflammation and fibrosis of the myocardium, although no changes were observed in the myocardial parasitism and parasitaemia rate [46]. This study suggests that CCL4/MIP-1 beta plays a role in preventing excessive inflammation rather than the control of parasite replication.

\section{DNA vaccines as immunotherapy against Chagas disease}

All antigens that have been used in the formulation of therapeutic DNA vaccines belong to the trans-sialidase family.

The Sanchez-Burgos group performed intraperitoneal infection in ICR mice with 500 parasites. Five and 12 days later mice were treated with $20 \mu \mathrm{g}$ of DNA plasmid containing theTSA-1, TS, ASP-2-like, Tc52 and Tc24 antigens. The DNA treatment with the gene encoding Tc52 reduced the parasitaemia and heart parasite load and improved the survival, but myocarditis was not affected significantly. The plasmids encoding Tc24 and TSA-1 significantly reduced parasitaemia, mortality, myocarditis and heart parasite load [47]. These data demonstrate that the therapeutic vaccination efficacy is dependent on the antigen and suggest that DNA vaccines encoding Tc24, TSA-1 and Tc52 represent good candidates for further studies for a therapeutic vaccine against Chagas disease.

Dumonteil et al. infected BALB/c mice with lethal doses of parasites and subsequently mice were treated with two immunizations of $100 \mu \mathrm{g}$ of DNA plasmid containing the Tc24 antigen, starting on day 5 postinfection. The treated animals showed parasitaemia and heart tissue inflammation reduction; the survival was more than $70 \%$ with TSA-1 and $100 \%$ with Tc24. Parasitological heart tissue analysis indicated that most of the mice contained parasite's kinetoplast DNA but a few of them showed living parasites, suggesting that the immunotherapy was effective but did not totally eliminate parasites [48]. In this strategy where the infection is already established, the use of therapeutic vaccines may help to reduce the risk of developing the acute stage of infection and therefore the associated pathology, but the parasite cannot be totally eliminated.

In a subsequent study, Zapata et al. investigated changes of $\mathrm{T}$ cell populations induced by DNA vaccine as immunotherapy. The ICR mice were infected with 500 blood trypomastigotes and treated during the acute or chronic stage with two doses of $100 \mu \mathrm{g}$ of DNA. Flow cytometry indicated that the therapeutic vaccine induced a rapid increase in the number of $\mathrm{CD}_{4}^{+}$and $\mathrm{CD} 8^{+} \mathrm{T}$ cells in both the acute and chronic stages. Also there was a rapid increase in IFN- $\gamma$ production by $\mathrm{CD}^{+} \mathrm{T}$ cells after treatment for the chronic phase. The effects of these changes in the infection control required long periods of time to be detectable but resulted in a myocarditis and parasite load reduction in both stages of the infection as tested by histopathological analysis and PCR semiquantitative detection of T.cruzi in cardiac tissue [49]. These results suggest that the DNA vaccines inducing $\mathrm{CD}^{+}{ }^{+} \mathrm{T}$ cell activity and IFN- $\gamma$ production, as Tc24 gene, might be good candidates for effective therapeutic vaccination against $T$. cruzi infection.

Aparicio-Burgos et al. induced an antigen specific IgM and IgG response (IgG2a, IgG1) in dogs after vaccination with TcVac1, which consisted of various plasmids encoding T. cruzi antigens, IL-12 and GM-CSF. Increased $\mathrm{CD}^{+} \mathrm{T}$ cell proliferation and IFN- $\gamma$ production as well as suppression of phagocyte activity, were observed. Parasitaemia was controlled and a moderate decrease in the triatomine infectivity was produced by the vaccination; however, heart parasite load and electrocardiographic and histopatologic alterations were not prevented but were less severe than in non-immunized dogs [50]. These results showed a moderate effect on both the acute and the chronic stages of infection but do not avoid the disease.

Rigato's group used a plasmid and a human adenovirus Type 5 (HuAd5) with a replication defect expressing the T. cruzi amastigote surface protein 2 (ASP-2). The aim was to elucidate the immmuno-protective memory $\mathrm{T}$ cell phenotype and function. Short and long-term $\mathrm{CD} 8^{+} \mathrm{T}$ cell populations available were compared in detail after immunization. It was found that despite the timing, both populations overlapped greatly with respect to functional and phenotypic characteristics [51]. In a subsequent study it was observed that the up-regulation of CD95 expression and the phenotype of the pro-apoptotic pathogen-specific $\mathrm{CD}^{+} \mathrm{T}$ cells expanded during infection were significantly reduced when the adenoviral vaccine was provided at the time of the infection. In parallel, mice vaccinated with adenovirus and subsequently infected had a stronger cell immune response mediated by CD8 T cells and survived an otherwise lethal infection. It was concluded that a suboptimal response of $\mathrm{CD}^{+} \mathrm{T}$ cells is associated with up-regulation of CD95 expression and a pro-apoptotic phenotype. Both situations can be blocked by the adenoviral vaccination [52].

Hoft et al. tested whether co-administration of a plasmid encoding IL-15 (pIL-15) and a plasmid encoding trans-sialidase (pTS) of Trypanosoma cruzi could improve the duration of protection, this co-administration did not significantly alter the $\mathrm{T}$ cell response or the protection shortly after immunization. However, mice vaccinated with both plasmids and challenged 6 months post immunization were significantly more protected than those who received only the gene of $T$. cruzi. Improved 
protection correlated with a significant increase of specific IFN $\gamma$-producing $\mathrm{T}$ cells against trans-sialidase [53]. It was shown here that IL-15 may have a significant effect as an adjuvant for induction of increased protection against $T$. cruzi.

Nogueira et al. used the yellow fever virus as a vector to immunize mice with the gene encoding the $T$. cruzi ASP-2. It was shown that the recombinant virus YF17D/ ENS1/Tc was capable of priming $\mathrm{CD}^{+} \mathrm{T}$ cells directed against the T. cruzi TEWETG1 epitope producing IFN- $\gamma$ after challenge [54]. This study shows that the use of viral formulations can be a good strategy to induce protective immune responses against pathogens.

\section{Conclusions}

DNA vaccines have provent o be effective in a variety of preclinical models. This technology has been adopted by many researchers as a strategy to develop a suitable vaccine against Chagas disease. It has explored various immunogens (genes encoding a variety of $T$. cruzi proteins), and routes of administration as well as the use of immunomodulators.

Although no vaccine has been able to prevent the infection, in some cases immunizations have induced partial protection; these studies provide data that proves the feasibility of preventive and therapeutic DNA vaccines to control the T. cruzi infection. However, a better understanding of the protective immune responses that can effectively stop parasite development and contribute to the understanding and development of an effective vaccine remains necessary.

Prophylactic vaccines in humans would be a very effective control method for Chagas disease, which would also be further improved if they were also used in the veterinary sectors.

The therapeutic use of DNA vaccines against Chagas disease could improve the effectiveness of current treatment and thus provide a better prognosis for the patient. These vaccines for therapeutic use have shown no adverse or toxic effects.

The prophylactic or therapeutic DNA vaccines have been shown to reduce the duration and signs of the acute stage, whereas in the chronic phase have avoided or lessened the severity of heart damage and thereby prolonged the patients' lifetime.

Prophylactic vaccines have failed so far to prevent parasitic infection; therefore, the current challenge is to identify the ideal antigen.

There is a need to create new formulations that can improve the existing protection achieved so far, as well as to test new methods of administration.

\section{Abbreviations}

CTL: Cytotoxic T lymphocytes; IL: Interleukin; GM-CSF: Granulocyte-macrophage colony-stimulating factor; Cz: Cruzipain; SCz: Salmonellacarrying a plasmid coding for cruzipain; rCz: recombinant cruzipain; Ig: Immunoglobulin; IFN-ү: Interferon-gamma; ASP: Amastigote surface protein; NK: Natural killer; a-GalCer: a-galactosylceramide; FCaBP: Flagellar $\mathrm{Ca}(2+)$ binding protein; TC $\beta 3$ : Homologue of the adaptin AP-3 complex $\beta 3$ subunit; NFkB: Nuclear factor kappa-light-chain-enhancer of activated B cells; MHC: Major histocompatibility complex; PFR: Paraflagelar rod protein; HSP70: 70 kilodalton heat shock protein; CCL4/MIP-1 beta: Chemokine (C-C mofif) ligand 4/macrophage inflammatory protein 1 beta; TNF: Tumor necrosis factor; CINVESTAV-IPN: Centro de Investigación y de Estudios Avanzados of the Instituto Politécnico Nacional; BIRMEX: Laboratorios de Biológicos y Reactivos de México, S. A. de C. V.; HuAd5: Human adenovirus type 5.

\section{Competing interests}

The authors declare that they have no competing interests.

\section{Authors' contributions}

MAF conceived and designed the study; MRC, SCCS and MCM drafted the "DNA vaccines", "DNA vaccines against Chagas disease" and "DNA vaccines as immunotherapy against Chagas disease" sections, respectively; ORM performed critical review of the contents of the entire article. MAF and ORM wrote the paper. All authors read and approved the final version of the review manuscript.

Received: 1 October 2014 Accepted: 13 February 2015

Published online: 24 February 2015

\section{References}

1. World Health Organization. Weekly epidemiological record. Geneve, Switzerland 2012;87:509-26. Available from: http://www.who.int/wer

2. Viotti R, Vigliano C, Armenti H, Segura E. Treatment of chronic Chagas' disease with benznidazole: clinical and serologic evolution of patients with long-term follow-up. Am Heart J. 1994;127:151-62.

3. Murcia L, Carrilero B, Segovia M. Limitations of currently available Chagas disease chemotherapy. Rev Esp Quimioter. 2012;25:1-3.

4. Astelbauer F, Walochnik J. Antiprotozoal compounds: state of the art and new developments. Int J Antimicrob Agents. 2011;38:118-24.

5. Urbina JA, Docampo R. Specific chemotherapy of Chagas disease: controversies and advances. Trends Parasitol. 2003;19:495-501.

6. Dumonteil E. DNA vaccines against protozoan parasites: opportunities and challenges. J Biomed Biotechnol. 2007;2007:90520.

7. Smirlis D, Soares MB. Selection of molecular targets for drug development against trypanosomatids. Subcell Biochem. 2014;74:43-76.

8. Soeiro MN, Werbovetz K, Boykin DW, Wilson WD, Wang MZ, Hemphill A. Novel amidines and analogues as promising agents against intracellular parasites: a systematic review. Parasitology. 2013;140:929-51.

9. Montgomery DL, Ulmer JB, Donnelly JJ, Liu MA. DNA Vaccines. Pharmacol Ther. 1997;74:195-205.

10. Coban C, Kobiyama K, Aoshi T, Takeshita F, Horii T, Akira S, et al. Novel strategies to improve DNA vaccine immunogenicity. Curr Gene Ther 2011:11:479-84.

11. Prompetchara E, Ketloy C, Keelapang P, Sittisombut N, Ruxrungtham K. Induction of Neutralizing Antibody Response against Four Dengue Viruses in Mice by Intramuscular Electroporation of Tetravalent DNA Vaccines. PLOS ONE. 2014;9:e92643.

12. Dutton JL, Li B, Woo WP, Marshak JO, Xu Y, Huang ML, et al. A novel DNA vaccine technology conveying protection against a lethal herpes simplex viral challenge in mice. PLOS ONE. 2013;8:e76407.

13. Obeng-Adjei N, Hutnick NA, Yan J, Chu JS, Myles DJ, Morrow MP, et al. DNA vaccine cocktail expressing genotype $A$ and $C$ HBV surface and consensus core antigens generates robust cytotoxic and antibody responses in mice and Rhesus macaques. Cancer Gene Ther. 2013;20:652-62.

14. Olgasi C, Dentelli P, Rosso A, lavello A, Togliatto G, Toto V, et al. DNA vaccination against membrane-bound Kit ligand: a new approach to inhibiting tumour growth and angiogenesis. Eur J Cancer. 2014;50:234-46.

15. Liu H, Geng S, Feng C, Xie X, Wu B, Chen X, et al. A DNA vaccine targeting p42.3 induces protective antitumor immunity via eliciting cytotoxic CD $8^{+} T$ lymphocytes in a murine melanoma model. Hum Vaccin Immunother. 2013:9:2196-202.

16. Ho PP, Fontoura P, Ruiz PJ, Steinman L, Garren H. An immunomodulatory $\mathrm{GpG}$ oligonucleotide for the treatment of autoimmunity via the innate and adaptive immune systems. J Immunol. 2003;171:4920-6. 
17. Wang Y, Wang YM, Wang Y, Zheng G, Zhang GY, Zhou JJ, et al. DNA vaccine encoding CD40 targeted to dendritic cells in situ prevents the development of Heymann nephritis in rats. Kidney Int. 2013;83:223-32.

18. Jégou JF, Chan P, Schouft MT, Gasque P, Vaudry H, Fontaine M. Protective DNA vaccination against myelin oligodendrocyte glycoprotein is overcome by C3d in experimental autoimmune encephalomyelitis. Mol Immunol. 2007:44:3691-701.

19. Weiss R, Scheiblhofer S, Thalhamer J. Allergens are not pathogens: Why immunization against allergy differs from vaccination against infectious diseases. Hum Vaccin Immunother. 2014;10:703-7.

20. Song LQ, Li Y, Li WN, Zhang W, Qi HW, Wu CG. Safety and immunogenicity of a DNA vaccine encoding human calcium-activated chloride channel 1 (hCLCA1) in asthmatic mice. Int Arch Allergy Immunol. 2013;161:243-51.

21. Pulsawat $P$, Pitakpolrat $P$, Prompetchara $E$, Kaewamatawong $T$, Techakriengkrai N, Sirivichayakul S, et al. Optimization of a Der p 2-based prophylactic DNA vaccine against house dust mite allergy. Immunol Lett. 2013;151:23-30

22. Wolff JA, Malone RW, Williams P, Chong W, Acsadi G, Jani A, et al. Direct gene transfer into mouse muscle in vivo. Science. 1990;247:1465-8.

23. Lew D, Parker SE, Latimer T, Abai AM, Kuwahara-Rundell A, Doh SG, et al. Cancer gene therapy using plasmid DNA: pharmacokinetic study of DNA following injection in mice. Hum Gene Ther. 1995;6:553-64.

24. Waine GJ, McManus DP. Nucleic acids: vaccines of the future. Parasitol Today. 1995;11:113-6.

25. Mairhofer J, Lara AR. Advances in host and vector development for the production of plasmid DNA vaccines. Methods Mol Biol. 2014;1139:505-41.

26. Hassan IA, Wang S, Xu L, Yan R, Song X, Li X. DNA vaccination with a gene encoding Toxoplasma gondii Deoxyribose Phosphate Aldolase (TgDPA) induces partial protective immunity against lethal challenge in mice. Parasit Vectors. 2014;7:431.

27. Cong H, Zhang M, Xin Q, Wang Z, Li Y, Zhao Q, et al. Compound DNA vaccine encoding SAG1/ SAG3 with A2/B subunit of cholera toxin as a geneticadjuvant protects BALB/C mice against Toxoplasma gondii. Parasit Vectors. 2013;6:63

28. Wang $X$, Zhang L, Chi Y, Hoellwarth J, Zhou S, Wen $X$, et al. The nature and combination of subunits used in epitope-based Schistosoma japonicum vaccine formulations affect their efficacy. Parasit Vectors. 2010;3:109.

29. Garg N, Tarleton R. Genetic immunization elicits antigen-specific protective immune responses and decreases disease severity in Trypanosoma cruzi infection. Infect Immun. 2002;70:5547-55.

30. Boscardin SB, Kinoshita SS, Fujimura AE, Rodrigues MM. Immunization with CDNA expressed by amastigotes of Trypanosoma cruzi elicits protective immune response against experimental infection. Infect Immun. 2003;71:2744-57.

31. Miyahira Y, Katae M, Takeda K, Yagita H, Okumura K, Kobayashi S, et al. Activation of natural killer $T$ cells by alpha-galactosylceramide impairs DNA vaccine-induced protective immunity against Trypanosoma cruzi. Infect Immun. 2003;71:1234-41.

32. Mussalem JS, Vasconcelos JR, Squaiella CC, Ananias RZ, Braga EG, Rodrigues $\mathrm{MM}$, et al. Adjuvant effect of the Propionibacterium acnes and its purified soluble polysaccharide on the immunization with plasmidial DNA containing a Trypanosoma cruzi gene. Microbiol Immunol. 2006;50:253-63.

33. Bhatia V, Garg NJ. Previously Unrecognized Vaccine Candidates Control Trypanosoma cruzi Infection and Immunopathology in Mice. Clin Vaccine Immunol. 2008;15:1158-64.

34. Dumonteil E, Bottazzi ME, Zhan B, Heffernan MJ, Jones K, Valenzuela JG, et al. Accelerating the development of a therapeutic vaccine for human Chagas disease: rationale and prospects. Expert Rev Vaccines. 2012;11:1043-55.

35. Salgado-Jiménez B, Arce-Fonseca M, Baylón-Pacheco L, Talamás-Rohana P, Rosales-Encina JL. Differential immune response in mice immunized with the A, R or C domain from TCSP protein of Trypanosoma cruzi or with the coding DNAs. Parasite Immunol. 2013;35:32-41.

36. Arce-Fonseca M, Ramos-Ligonio A, López-Monteón A, Salgado-Jiménez B, Talamás-Rohana P, Rosales-Encina JL. A DNA vaccine encoding for TcSSP4 induces protection against acute and chronic infection in experimental Chagas disease. Int J Biol Sci. 2011;7:1230-8.

37. Arce-Fonseca M, Ballinas-Verdugo MA, Zenteno ER, Suárez-Flores D, Carrillo-Sánchez SC, Alejandre-Aguilar R, et al. Specific humoral and cellular immunity induced by Trypanosoma cruzi DNA immunization in a canine model. Vet Res. 2013;44:15.
38. Rodríguez-Morales O, Pérez-Leyva MM, Ballinas-Verdugo MA, Carrillo-Sánchez SC, Rosales-Encina JL, Alejandre-Aguilar R, et al. Plasmid DNA immunization with Trypanosoma cruzi genes induces cardiac and clinical protection against Chagas disease in the canine model. Vet Res. 2012;43:79.

39. Rodríguez-Morales O, Carrillo-Sánchez SC, García-Mendoza H, Aranda-Fraustro A, Ballinas-Verdugo MA, Alejandre-Aguilar R, et al. Effect of the plasmid-DNA vaccination on macroscopic and microscopic damage caused by the experimental chronic Trypanosoma cruzi infection in the canine model. Biomed Res Int. 2013;2013:826570.

40. Schnapp AR, Eickhoff CS, Scharfstein J, Hoft DF. Induction of B- and T-cell respondes to cruzipain in the murine model of Trypanosoma cruzi infection. Microbes Infect. 2002;4:805-13.

41. Cazorla SI, Becker PD, Frank FM, Ebensen T, Sartori MJ, Corral RS, et al. Oral vaccination with Salmonella enterica as a cruzipain-DNA delivery system confers protective immunity against Trypanosoma cruzi. Infect Immun. 2008;76:324-33.

42. Fralish BH, Tarleton RL. Genetic immunization with LYT1 or a pool of trans-sialidase genes protects mice from lethal Trypanosoma cruzi infection Vaccine. 2003;21:3070-80

43. Miyahira T, Takashima Y, Kobayashi S, Matsumoto Y, Takeuchi T, Ohyanagi-Hara $M$, et al. Immune response against a single CD8+ - T cell epitope induced by virus vector vaccination can successfully control Trypanosoma cruzi infection. Infect Immun. 2005;73:7356-65.

44. García GA, Arnaiz MR, Laucella SA, Esteva MI, Ainciart N, Riarte A, et al Immunological and pathological responses in BALB/C mice induced by genetic administration of Tc 13 antigen of Trypanosoma cruzi. Parasitology. 2006;132:855-66

45. Morell M, Thomas MC, Caballero T, Alonso C, López MC. The genetic immunization with paraflagellar rod protein-2 fused to the HSP70 confers protection against late Trypanosoma cruzi infection. Vaccine. 2006;24:7046-55.

46. Roffe E, Souza AL, Caetano BC, Machado PP, Barcelos LS, Russo RC, et al. A DNA vaccine encoding CCL4/MIP-1 beta enhances myocarditis in experimental Trypanosoma cruzi infection in rats. Microbes Infect. 2006:8:2745-55.

47. Sanchez-Burgos G, Mezquita-Vega RG, Escobedo-Ortegon J, Ramirez-Sierra MJ, Arjona-Torres A, Ouaissi A, et al. Comparative evalutation of therapeutic DNA vaccines against Trypanosoma cruzi in mice. FEMS Immunol Med Microbiol. 2007:50:333-41.

48. Dumonteil E, Escobedo-Ortegon J, Reyes-Rodriguez N, Arjona-Torres A Ramirez-Sierra MJ. Immunotherapy of Trypanosoma cruzi infection with DNA vaccines in mice. Infect Immun. 2004;72:46-53.

49. Zapata-Estrella H, Hummel-Newell C, Sanchez-Burgos G, Escobedo-Ortegon J, Ramirez-Sierra MJ, Arjona-Torres A, et al. Control of Trypanosoma cruzi infection and changes in T-cell populations induced by a therapeutic DNA vaccine in mice. Immunol Lett. 2006;103:186-91.

50. Aparicio-Burgos JE, Ochoa-García L, Zepeda-Escobar JA, Gupta S, Dhiman M, Martínez JS, et al. Testing the efficacy of a multi-component DNA-prime/ DNA-boost vaccine against Trypanosoma cruzi infection in dogs. PLoS Negl Trop Dis. 2011;5:e1050

51. Rigato PO, de Alencar BC, de Vasconcelos JR, Dominguez MR, Araújo AF, Machado AV, et al. Heterologous plasmid DNA prime-recombinant human adenovirus 5 boost vaccination generates a stable pool of protective long-lived CD8(+) T effector memory cells specific for a human parasite, Trypanosoma cruzi. Infect Immun. 2011;79:2120-30.

52. Vasconcelos JR, Bruña-Romero O, Araújo AF, Dominguez MR, Ersching J, de Alencar BC. Pathogen-induced proapoptotic phenotype and high CD95 (Fas) expression accompany a suboptimal CD8+ T-cell response: reversal by adenoviral vaccine. PLoS Pathog. 2012;8:e1002699.

53. Eickhoff CS, Vasconcelos JR, Sullivan NL, Blazevic A, Bruna-Romero O, Rodrigues MM, et al. Co-administration of a plasmid DNA encoding IL-15 improves long-term protection of a genetic vaccine against Trypanosoma cruzi. PLoS Negl Trop Dis. 2011;5:e983.

54. Nogueira RT, Nogueira AR, Pereira MC, Rodrigues MM, Neves PC, Galler R, et al. Recombinant yellow fever viruses elicit CD8+ T cell responses and protective immunity against Trypanosoma cruzi. PLoS ONE. 2013;8:e59347. 